\title{
Automation is just beginning
}

\section{The laboratory application of computers is being extended rapidly, but there is some way to go before the penetration of these machines is as complete as, say, that of the Bunsen burner.}

A HEW years from now, the heading on these pages will be entirely inappropriate. The idea that the use of computers in laboratories is sufficiently novel to be remarked upon will have ceased entirely to be noteworthy, just as it would now seem strange to assemble a few pages of this journal under the heading "oscilloscopes in the laboratory". So charitable readers are invited to regard the pages that follow as a reminder that the present is a transitional period, one in which it is not yet second nature for experimentalists and theorists alike to use all the tools now potentially at their disposal.

It is also worth remarking that the present hectic period during which the laboratory computer is being made familiar has its roots in a period which long predates the development of electronic computing machinery. One of the obvious illustrations of this truth is in the development early this century by C.T.R. Wilson of the cloud chamber, first for stimulating processes occurring in the atmosphere, later for the recording of cosmic ray particles by the trail of droplets produced in a supersaturated vapour. The first studies of cosmic rays were necessarily made with cloud chambers whose expansion (to give supersaturation) was carried out at random. But the technique became productive only when, in the 1920 s, cloud chambers could be made to record events only when the chance of something interesting being observed was high.

The (Geiger) counter-controlled cloud chambers of those days are forerunners of the computer-controlled experimental rigs that are now commonplace in many important ways, but not least because they stimulated the development (with thermionic valves or tubes) of the logic circuits now embodied by the thousands in microprocessors. Over half a century, the practice of particle physics has been inextricably bound up with the development of electronic techniques for making sure that observations are made only when it is profitable to do so. And while the techniques have now spread to a host of other fields in which the objective is to record transitory phenomena, accelerator physics remains the citadel of the selfconscious practice of selecting from a mass of naturally arising happenings only those about which observational data are likely to be useful.

Computer-controlled experiment has the virtue of economizing in effort in the way most likely to be productive - by the avoidance of unwanted data. This is the spirit in which, in many biological applications (and more generally), it is possible to record data only for as long as necessary to provide some predetermined degree of statistical certainty. The prerequisite of such procedures is that there should be a computing facility "on-line" with the data-gathering equipment, carrying out an analysis as the experiments proceeds. Again, inevitably, the accelerator people have blazed a trail which everybody will soon be compelled to follow. For the collection of needless data is not merely inelegant but an encumbrance, a distraction. Even now, however, the point is not sufficiently forcefully made in teaching laboratories.

The notion that computers in one form or another have an important part to play in the collection ("logging") and analysis of data is, by comparison, more readily accepted. What seems to be less widely appreciated is that there are already a great many kinds of investigations now commonly undertaken that would be beyond the scope (and stamina) of people without the assistance of computing machinery. Particle physics has again been the pathfinder in this trend, with its techniques for the recording of tracks (or points along the tracks) left by particles in detectors of various kinds, and for their subsequent automatic analysis.

Inevitably, this practice has spread like wildfire. Deep seismic refraction, both in the exploration for petroleum and in geophysical investigations, generates such masses of data that only computer recording and analysis is feasible. The identification as well as the measurement of stellar and galactic objects on photographic plates collected by astronomers is similarly a way of carrying out tasks that would otherwise be impracticable. And even where the quantities of data are not nearly as overwhelming, there remains the possibility that they will not be fully interpreted because the investigator has not the stomach for the task or, more charitably, takes the view that his priorities lie elsewhere. Yet even in this uncontroversial field, where the value and the objectivity of automatic data analysis is generally accepted, teaching laboratories are not nearly as well equipped as they might be.

The application of computers as means of retrieving data is, by comparison, commonly undervalued. Many people have found the widely available bibliographical databases less than a substitute for the professional literature. And it is true that many of these systems are needlessly wooden and inflexible. There may be more promise in the schemes (some of which are referred to in the pages that follow) that assist people to construct their own individual databases, for mnemonics are almost certain to be more "friendly" (in the computer jargon) than substitutes for one's memory.

The use of databases simply for the recognition of meaningful portions of the nucleotide sequences of DNA which have become a common feature of the literature is perhaps the most telling illustration. The plain truth is that it is almost conceptually impossible for a person to compare the sequence of some nucleotide sequence he has constructed with a library of known gene sequences so as to pick out those which most closely resemble each other. Not so long ago, an author of an article submitted for publication was much chagrined that a referee with access to a better computer program was able to spot a similarity that had previously gone unrecognized. But this is only one set of circumstances in many where the full power of computer retrieval remains to be recognized. Rivalry between laboratories will no doubt bring other cases to light.

But none of this, it will be remarked, bears on what must seem the most obvious application of computers in the laboratory - the accomplishment of mathematical tasks that would otherwise be beyond the scope of ordinary people. The use of powerful computers for the simulation of, say, the evolution of weather systems requires no special justification, but the huge installations by which these tasks are accomplished are (and are likely to remain) the exceptions, not the rule.

More interesting are the applications of computer programs to ordinary tedious tasks of algebraic analysis, still regarded as a kind of frivolity or luxury, and to fields in which the underlying characteristics of a mathematical process would not otherwise be apparent. Who would at this stage claim that the importance of chaotic (or "deterministically random") phenomena would have been widely recognized without the computer simulations of the process that are now commonplace? Or that the study of fractal systems could have been accomplished analytically if only mathematicians were less idle? 